Original Research Paper

\title{
Continuity Function on Partial Metric Space
}

\author{
${ }^{1}$ Fitri Aryani, ${ }^{1}$ Hafiz Mahmud, ${ }^{1}$ Corry Corazon Marzuki, \\ ${ }^{1}$ Mohammad Soleh, ${ }^{1}$ Rado Yendra and ${ }^{2}$ Ahmad Fudholi \\ ${ }^{I}$ Department of Mathematics, Faculty of Science and Technology, \\ Universitas Islam Negeri Sultan SyarifKasim (UIN Suska) 28293, Pekanbaru, Riau, Indonesia \\ ${ }^{2}$ Solar Energy Research Institute, UniversitiKebangsaan Malaysia, 43600 Bangi Selangor, Malaysia
}

\author{
Article history \\ Received: 27-08-2016 \\ Revised: 29-10-2016 \\ Accepted: 11-11-2016 \\ Corresponding Author: \\ Fitri Aryani \\ Department of Mathematics, \\ Faculty of Science and \\ Technology, UIN Suska, \\ Pekanbaru, Riau, Indonesia; \\ Email: a.fudholi@gmail.com \\ khodijah_fitri@uin-suska.ac.id
}

\section{Introduction}

Lately, there has been a lot of mathematicians interested in developing the study of metric spaces that appear various generalization of metric spaces. One generalization of a metric space is a partial metric space developed by Matthews in 1992. Several studies on partial metric space has ever done is Romaguera and Schellekens (2005) researching on the basic concept of a partial metric space in the Quantitative Domain Theory in journals titled "Partial monoids and Semivaluation Metric Spaces". Subsequently, Wahyuni (2012) in the journal entitled "Topology of Partial Metric" examines the topology built by base ball open partial metric.

Subsequently, in 2014 Devi Arintika have discussed about Banach fixed point theorem applicable on a partial metric spaces in his journal, entitled "Generalitation Banach Fix Point Theorem on Partial Metric Space". Then, 2015 Ge Xun and Lin Shou examines the existence and uniqueness theorems for completion of a partial metric space in the journal entitled "Completion of Partial Metric Spaces".

Based on these reviews, the authors are interested to observe about one generalizations of metric space that is partial metric space and concepts of continuity of partial metric space. The partial metric space will be observed in this study is:

$$
p(x, y)=\frac{|x-y|+|x|+|y|}{2}
$$

\section{Preliminaries}

\section{Metric and Metric Space}

Definition 1. (Thomson et al., 2008)

Let $S$ is nonempty set. A function:

$$
d: S \times S \rightarrow \mathbb{R}
$$

Called metric if it satisfies the following 4 properties:

- $\quad d(x, y) \geq 0$, for all $x, y \in S$

- $d(x, y)=0$, if and only if $x=y$

- $d(x, y)=d(y, x)$ for all $x, y \in S$

- $\quad d(x, y) \leq d(x, z)+d(z, y)$ for all $x, y, z \in S$

If $d$ is metric on nonempty set $S$, then $S$ called metric space, as described in the following definition:

Definition 2. (Thomson et al., 2008)

Metric space is a set of pairs $(S, d)$, where $S$ is nonempty set and $d$ is a metric on $S$.

Definition metric and metric space more easily understood by observing the following example:

\section{Example 1}

Let a nonempty set $S=\mathbb{R}$ with function $d: S \times S \rightarrow \mathbb{R}$ where $d(x, y)=|x-y|$ for all $x, y \in S$. Show that $(S, d)$ is a metric space! 


\section{Solution}

Given a nonempty set $S=\mathbb{R}$ with $d: S \times S \rightarrow \mathbb{R}$ where $d(x, y)=|x-y|$ for all $x, y \in S$, will be shown that $(S, d)$ is metric space, means nonempty $S$ with function $d: S \times S \rightarrow \mathbb{R}$ where $d(x, y)=|x-y|$ for all $x, y \in S$ must following four properties from Definition 1. Properties $(a),(b),(c)$ already clearly fulfilled for $(x, y)=|x-y|$, while for properties $(d)$ can be provided by using the triangleinequality in absolute value. Consider that:

$$
\begin{aligned}
& d(x, y)=|x-y|=|(x-z)+(z-y)| \\
& \leq|x-z|+|z-y| \\
& =d(x, z)+d(z, y) \text { for all } x, y, z \epsilon S
\end{aligned}
$$

So $(S, d)$ is metric space.

\section{Partial Metric and Partial Metric Space}

Partial metric space is a generalization of metric spaces. Consider the following definitions:

\section{Definition 3. (Romaguera and Schellenkens, 2005)}

A partial metric on nonempty set $S$ is a function $p: S \times S \rightarrow \mathbb{R}^{+}$, such that for every $x, y, z \in S$ satisfy the following axioms:

- $p(x, x)=p(x, y)=p(y, y)$ if and only if $x=y$

- $p(x, x) \leq p(y, x)$

- $p(x, y)=p(y, x)$

- $p(x, z) \leq p(x, y)+p(y, z)-p(y, y)$

According to Malhotra et al. (2014) says that a partial metric space is a pair $(S, d)$ such that $S$ is nonempty set and $p$ is metric partial.

The function on the partial metric is a generalization of the axiom minimal metrics such that for every $x \in S$, $d(x, x)$ does not need to be zero, in other terms is known as nonzero self-distance. Axiom obtained from the generalization is following properties $p(x, x) \leq p(y, x)$ (Wahyuni, 2012). Definition of partial metrics and partial metric spaces more easily understood by observing the following example:

\section{Example 2}

Let $S=\mathbb{R}$. Defined functions $p: \mathbb{R} \times \mathbb{R} \rightarrow \mathbb{R}^{+}$as follows:

$$
p(x, y)=\frac{|x-y|+|x|+|y|}{2}
$$

$\forall x, y \in \mathbb{R}$. Show that $(S, p)$ is partial metric space.

\section{Solution}

Given a nonempty set $S=\mathbb{R}$ with $p: \mathbb{R} \times \mathbb{R} \rightarrow \mathbb{R}^{+}$where $p(x, y)=\frac{|x-y|+|x|+|y|}{2}$, for all $x, y \in \mathbb{R}$ will be shown that $(S, d)$ is partial metric space, means nonempty $S$ with function $p: \mathbb{R} \times \mathbb{R} \rightarrow \mathbb{R}^{+}$where $p(x, y)=\frac{|x-y|+|x|+|y|}{2}$, for all $x, y, z \in \mathbb{R}$ must following four properties from Definition 3. Properties (i), (ii), (iii) and (iv) already clearly fulfilled for $p(x, y)=\frac{|x-y|+|x|+|y|}{2}$, so $(S, p)$ is partial metric space.

Furthermore, the definition will be given a sequence converging on a partial metric space.

Definition 4. (Arintika, 2012)

A sequence $\left\{x_{n}\right\}$ in partial metric space $(S, p)$ convergent to $c \in S$, for each $\varepsilon>0$ there $N \in \mathbb{N}$ such that for all $n \geq N$ satisfy:

$$
p\left(x_{n}, c\right)-p(c, c)<\varepsilon
$$

and:

$$
p\left(x_{n}, c\right)-p\left(x_{n}, x_{n}\right)<\varepsilon
$$

\section{Main Results}

\section{Continuous Function on Partial Metric Space}

Before defining the concepts of continuous functions on a metric space partial, will be determined beforehand definition of the partial metric subspace, which is as follows:

\section{Definition 5}

If $(S, p)$ is partial metric space and $A \subseteq S$, then $(A, p)$ called partial metric subspace $(S, p)$.

The following definitions will explain the function which is continuous of partial metric space at a point.

\section{Definition 6}

Let $\left(S_{1}, p\right)$ and $\left(S_{2}, p\right)$ is partial metric space, $A \subseteq S_{1}$, function $f: A \rightarrow S_{2}$ and $c \in A$. Function $f$ continuous at $c$, if for any $\varepsilon>0$ there is $\delta>0$ such that if $x \in A$ :

$$
p(x, c)-p(x, x)<\delta
$$

and:

$$
p(x, c)-p(c, c)<\delta
$$


then:

$$
p(f(x), f(c))-p(f(x), f(x))<\varepsilon
$$

and:

$$
p(f(x), f(c))-p(f(c), f(c))<\varepsilon
$$

\section{Theorem 1}

Let $\left(S_{1}, p\right)$ and $\left(S_{2}, p\right)$ arepartial metric space and $A \subseteq S_{1}$ If $f: A \rightarrow S_{2}$ and $c$ is limit point of $A$, then $f$ only have onelimit point at $c$ (uniqueness of limits).

\section{Proof}

Let $L$ and $L^{\prime}$ satisfyDefinition 6, show that $L=L^{\prime}$.

If $\varepsilon>0$ is given, there $\delta^{\prime}(\varepsilon / 2)>0$ such that if for all $x \in A$ :

$$
0<p(x, c)-p(x, x)<\delta^{\prime}(\varepsilon / 2)
$$

and:

$$
0<p(x, c)-p(c, c)<\delta^{\prime}(\varepsilon / 2)
$$

then:

$$
p(f(x), L)-p(f(x), f(x))<\varepsilon / 2
$$

and:

$$
p(f(x), L)-p(L, L)<\varepsilon / 2
$$

Then, there exist $\delta^{\prime \prime}(\varepsilon / 2)>0$ such that if $x \in A$ :

$$
0<p(x, c)-p(x, x)<\delta^{\prime \prime}(\varepsilon / 2)
$$

and:

$$
0<p(x, c)-p(c, c)<\delta^{\prime \prime}(\varepsilon / 2)
$$

then:

$$
p\left(f(x), L^{\prime}\right)-p(f(x), f(x))<\varepsilon / 2
$$

and:

$$
p\left(f(x), L^{\prime}\right)-p\left(L^{\prime}, L^{\prime}\right)<\varepsilon / 2
$$

Chosen $\delta=\min \left\{\delta^{\prime}(\varepsilon / 2), \delta^{\prime \prime}(\varepsilon / 2)\right\}$, if $x \in A$ :

$$
0<p(x, c)-p(x, x)<\delta
$$

and:

$$
0<p(x, c)-p(c, c)<\delta
$$

We have:

$$
\begin{aligned}
& p(f(x), L)-p(f(x), f(x))<\varepsilon / 2 \\
& p(f(x), L)-p(L, L)<\varepsilon / 2
\end{aligned}
$$

and:

$$
\begin{aligned}
& p\left(f(x), L^{\prime}\right)-p(f(x), f(x))<\varepsilon / 2 \\
& p\left(f(x), L^{\prime}\right)-p\left(L^{\prime}, L^{\prime}\right)<\varepsilon / 2
\end{aligned}
$$

\section{Consequently:}

$$
\begin{aligned}
& p\left(L, L^{\prime}\right)-p(L, L) \\
\leq & \left(p(L, f(x))+p\left(f(x), L^{\prime}\right)-p(f(x), f(x))\right)-p(L, L) \\
= & p(f(x), L)+p\left(f(x), L^{\prime}\right)-p(f(x), f(x))-p(L, L) \\
= & p(f(x), L)-p(L, L)+p\left(f(x), L^{\prime}\right)-p(f(x), f(x)) \\
< & \frac{\varepsilon}{2}+\frac{\varepsilon}{2} \\
= & \varepsilon
\end{aligned}
$$

and:

$$
\begin{aligned}
& p\left(L, L^{\prime}\right)-p\left(L^{\prime}, L^{\prime}\right) \\
\leq & \left(p(L, f(x))+p\left(f(x), L^{\prime}\right)-p(f(x), f(x))\right)-p\left(L^{\prime}, L^{\prime}\right) \\
= & p(f(x), L)+p\left(f(x), L^{\prime}\right)-p(f(x), f(x))-p\left(L^{\prime}, L^{\prime}\right) \\
= & p(f(x), L)-p(f(x), f(x))+p\left(f(x), L^{\prime}\right)-p\left(L^{\prime}, L^{\prime}\right) \\
< & \frac{\varepsilon}{2}+\frac{\varepsilon}{2} \\
= & \varepsilon .
\end{aligned}
$$

Since $\varepsilon>0$ is an arbitary, then we have:

$$
p\left(L, L^{\prime}\right)-p(L, L)=0
$$

and:

$$
p\left(L, L^{\prime}\right)-p\left(L^{\prime}, L^{\prime}\right)=0
$$

Such that:

$$
p\left(L, L^{\prime}\right)=p(L, L)=p\left(L^{\prime}, L^{\prime}\right)
$$

Basedon Definition 3 axiom $(i)$ then $\mathrm{L}=L^{\prime}$. 
Proof that $f$ only have one limit point at $c$ (uniqueness of limits).

After we get concepts ofthe limit of a functionon partial metric space, then we will observe about concepts of continuous functions on partial metric space. The following definition explain about continuous function on partial metric space at a point.

\section{Definition 7}

Let $\left(S_{1}, p\right)$ and $\left(S_{2}, p\right)$ arepartial metric space, $A \subseteq S_{1}$, function $f: A \rightarrow S_{2}$ and $c \in A$. $f$ continueousat $c$, if for each $\varepsilon>0$ there exist $\delta>0$ such that if $x \in A$ :

$$
p(x, c)-p(x, x)<\delta
$$

and:

$$
p(x, c)-p(c, c)<\delta
$$

then:

$$
p(f(x), f(c))-p(f(x), f(x))<\varepsilon
$$

and:

$$
p(f(x), f(c))-p(f(c), f(c))<\varepsilon
$$

To understand continuity of partial metric spaces, will be given in the following example:

\section{Example 3}

Given $\left(S_{1}, p\right)$ and $\left(S_{2}, p\right)$ is partial metric space and $A \subseteq S_{1}$. Show that function $f: A \rightarrow S_{2}$ defined on $A$ with $f(x)$ $=x$ is continues.

\section{Solution}

Given $f(x)=x$, will be shown for any $\varepsilon>0$ there is $\delta$ $>0$ such that if $x \in A$ :

$$
p(x, c)-p(x, x)<\delta
$$

and:

$$
p(x, c)-p(c, c)<\delta
$$

then:

$$
p(x, c)-p(x, x)<\varepsilon
$$

and:

$$
p(x, c)-p(c, c)<\varepsilon
$$

If $\varepsilon>0$ is given, choose $\delta=0$. Consider that if $x \in A$ :

$$
p(x, c)-p(x, x)<\delta
$$

and:

$$
p(x, c)-p(c, c)<\delta
$$

then:

$$
\begin{aligned}
& p(f(x), f(c))-p(f(x), f(x)) \\
& =p(x, c)-p(x, x) \\
& <\partial=\varepsilon,
\end{aligned}
$$

and:

$$
\begin{aligned}
& p(f(x), f(c))-p(f(c), f(c)) \\
& =p(x, c)-p(c, c) \\
& <\partial=\varepsilon .
\end{aligned}
$$

Therefore $f(x)=x$ is continuous on $A$.

Next, will be given a theorem about the function which is continuous in a sequence on partial metric space.

\section{Theorem 2}

Let $\left(S_{1}, p\right)$ and $\left(S_{2}, p\right)$ is partial metric space, $A \subseteq S_{1}$, $c \in A$ and function $f: A \rightarrow S_{2}$. The following statement is equivalent:

- $f$ continous at $c$

- For any sequence $\left\{x_{n}\right\}$ at $A$ convergent to $c \in A$, then sequence $\left\{f\left(x_{n}\right)\right\}$ convergent to $f(c)$

\section{Proof}

$(a) \Rightarrow(b)$ Given for any $\varepsilon>0$ there is $\delta>0$ such that if $x \in A$ :

$$
p(x, c)-p(x, x)<\delta
$$

and:

$$
p(x, c)-p(c, c)<\delta
$$

then:

$$
p(f(x), f(c))-p(f(x), f(x))<\varepsilon
$$

and:

$$
p(f(x), f(c))-p(f(c), f(c))<\varepsilon
$$


Let $\left\{x_{n}\right\}$ convergent to $c$, so base on Definition 4 , for $\delta>0$ there is $N \in \mathbb{N}$ such that $n \geq N$ be obtained:

$$
p\left(x_{n}, c\right)-p\left(x_{n}, x_{n}\right)<\delta
$$

and:

$$
p\left(x_{n}, c\right)-p(c, c)<\delta
$$

Therefore for all $n \geq N$ satisfy:

$$
p\left(f\left(x_{n}\right), f(c)\right)-p\left(f\left(x_{n}\right), f\left(x_{n}\right)\right)<\varepsilon
$$

and:

$$
p\left(f\left(x_{n}\right), f(c)\right)-p(f(c), f(c))<\varepsilon
$$

This shows that $\left\{f\left(x_{n}\right)\right\}$ convergent to $f(c)$.

$(b) \Rightarrow(a)$ Given that for any sequence $\left\{x_{n}\right\}$ at $A$ converging to $c \in A$, then sequence $\left\{f\left(x_{n}\right)\right\}$ convergent to $f(c)$ and will be shown that $f$ continous at $c$.

Suppose $f$ is not continuous at $c$, means $f$ is not continuous at $c$, like there $\varepsilon>0$ such that for any $\delta>0$ there is $x \in A$ satisfies:

$$
p(x, c)-p(x, x)<\delta
$$

and:

$$
p(x, c)-p(c, c)<\delta
$$

but:

$$
p(f(x), f(c))-p(f(x), f(x)) \geq \varepsilon
$$

or:

$$
p(f(x), f(c))-p(f(c), f(c)) \geq \varepsilon
$$

Chosen $\delta=\frac{1}{n}>0$ for each $n \in \mathbb{N}$ such that a sequence $\left\{x_{n}\right\} \in A$ satisfies:

$$
p\left(x_{n}, c\right)-p\left(x_{n}, x_{n}\right)<\frac{1}{n}
$$

and:

$$
p\left(x_{n}, c\right)-p(c, c)<\frac{1}{n}
$$

but:

$$
p\left(f\left(x_{n}\right), f(c)\right)-p\left(f\left(x_{n}\right), f\left(x_{n}\right)\right) \geq \varepsilon
$$

or:

$$
p\left(f\left(x_{n}\right), f(c)\right)-p(f(c), f(c)) \geq \varepsilon
$$

It means $\left\{x_{n}\right\}$ convergent to $c$, but $\left\{f\left(x_{n}\right)\right\}$ does not convergent to $f(c)$. This is a contradiction with $\left\{f\left(x_{n}\right)\right\}$ convergent to $f(c)$ and we conclude that $f$ continuous at $c$.

Next, given definition of a bounded sequence on partial metric space.

\section{Definition 8}

A sequence $X=\left\{x_{n}\right\}$ on partial metric space is bounded if there exist $M>0$ such that:

$$
\left|x_{n}\right| \leq M
$$

for all $n \in \mathbb{N}$.

Sequence convergent of partial metric space is limited, as described in the following theorem:

\section{Theorem 3.}

A sequence $\left\{x_{n}\right\}$ convergent of partial metric space is bounded.

\section{Proof}

If convergent sequence $\left\{x_{n}\right\}$ is given, let convergent to $c \in S$. Based on Definition 4, means for any $\varepsilon>0$ there is $N \in \mathbb{N}$ such that $n \geq N$ apply:

$$
p\left(x_{n}, c\right)-p(c, c)<\varepsilon
$$

and:

$$
p\left(x_{n}, c\right)-p\left(x_{n}, x_{n}\right)<\varepsilon
$$

Let $\varepsilon=1$, so:

$$
p\left(x_{n}, c\right)-p(c, c)<1
$$

and:

$$
p\left(x_{n}, c\right)-p\left(x_{n}, x_{n}\right)<1
$$

Obtained $\left|x_{n}\right|<1+|c|$, then select:

$$
M=\sup \left\{\left|x_{1}\right|,\left|x_{2}\right|, \cdots,\left|x_{N-1}\right|, 1+|c|\right\}
$$

This shows that $M>0$ such that:

$$
\left|x_{n}\right| \leq M
$$




\section{for each $n \in \mathbb{N}$.}

Therefore, sequence $\left\{x_{n}\right\}$ is Bounded.

\section{Conclusion}

Based on the main results, obtained some conclusions as follows:

- If $(S, p)$ is partial metric space and $A \subseteq S$, then $(A, p)$ is called partial metric subspace $(S, p)$

- Some of the concept of continuity function on partial metric space is as follows:

- $\quad$ Let $\left(S_{1}, p\right)$ and $\left(S_{2}, p\right)$ arepartial metric space and $A \subseteq S_{1}$. If $f: A \rightarrow S_{2}$ and $c$ is limit point of $A$, then $f$ only have onelimit point at $c$ (uniqueness of limits)

- Let $\left(S_{1}, p\right)$ and $\left(S_{2}, p\right)$ is partial metric space, $A \subseteq S_{1}, c \in A$ and function $f: A \rightarrow S_{2}$. The following statement is equivalent:

- $f$ continous at $c$

- For any sequence $\left\{x_{n}\right\}$ at $A$ convergent to $c \in A$, then sequence $\left\{f\left(x_{n}\right)\right\}$ convergent to $f(c)$

- A sequence $\left\{x_{n}\right\}$ convergent of partial metric space is bounded

\section{Acknowledgement}

The authors would like to thank the Faculty of Science and Technology, Universitas Islam Negeri Sultan SyarifKasim (UIN Suska) Pekanbaru, Riau, Indonesia for support.

\section{Author's Contributions}

Fitri Aryani: Design concept and paper writing of the paper.

Hafiz Mahmud: Literature review and paper writing of the paper

Corry Corazon Marzuki and Mohammad Soleh: Developed concept and analysis.

Rado Yendra: Revise and improve the final drafts of the paper.

Ahmad Fudholi: Critical review of the scientific content of the paper. Revise, improve and editing the final drafts of the paper.

\section{Ethics}

This article is original and contains unpublished material. The corresponding author confirms that all of the other authors have read and approved the manuscript and no ethical issues involved.

\section{References}

Arintika, D., 2012. Perluasan teorema titik tetap banachpada ruang metrik parsial. J. Matematika Univ. Brawijaya.

Malhotra, S.K., S. Radenovic and S. Shukla, 2014. Some fixed points results without monotone property in partially ordered metric-like spaces. J. Egypt. Math. Society, 22: 83-89.

DOI: 10.1016/j.joems.2013.06.010

Romaguera, S. and M. Schellenkens, 2005. Partial metric monoids and semivaluation spaces. Topol. Applic., 153: 948-962. DOI: 10.1016/j.topol.2005.01.023

Thomson, B.S., A.M. Bruckner and A.M. Bruckner, 2008. Elementary Real Analysis. 1st Edn., Prentice Hall, New York.

Wahyuni, D., 2012. Topologi metrik parsial, J. Matematika Unand, 1: 71-78. 